\title{
Insatisfação corporal e maturação biológica em atletas do sexo masculino
}

CDD. 20.ed. 152

796.033

\author{
Leonardo de Sousa FORTES* \\ Sebastião Sousa ALMEIDA** \\ Maria Elisa Caputo FERREIRA*
}

*Faculdade de Educação Física e Desportos, Universidade Federal de Juiz de Fora. ${ }^{* *}$ Faculdade de Filosofia, Ciências e Letras de Ribeirão Preto, Universidade de São Paulo.

\section{Resumo}

0 objetivo do presente estudo foi investigar a influência da maturação biológica na insatisfação corporal (IC) de jovens atletas do sexo masculino. Participaram da pesquisa 464 esportistas. A IC foi mensurada pelo Body Shape Questionnaire (BSO). A maturidade biológica foi estimada pela maturação somática. Peso, estatura e dobras cutâneas foram aferidos para calcular o índice de massa corporal (IMC) e estimar o percentual de gordura, respectivamente. Foram conduzidos testes estatísticos em "software" especializado para analisar os dados. Os resultados não evidenciaram diferenças estatisticamente significativas para as pontuações do BSO segundo classificações de maturação somática. Por outro lado, identificaram-se diferenças de IMC e percentual de gordura entre estágios maturacionais $(p<0,05)$. Deste modo, concluiuse que a IC dos jovens atletas não sofreu influência da maturação biológica.

PalaVRAS-CHAVE: Imagem corporal; Atletas; Esporte.

\section{Introdução}

Imagem corporal é um construto multidimensional que envolve fatores afetivos, cognitivos, comportamentais e sociais ${ }^{1}$. Segundo FiLAIRE et al. ${ }^{2}$, a imagem corporal possui duas dimensóes: perceptiva e atitudinal. A última estaria relacionada a crenças, pensamentos e sentimentos associados ao próprio corpo, por exemplo, a insatisfação corporal. Esta, por sua vez, diz respeito ao descontentamento que o indivíduo apresenta com o peso e a aparência física ${ }^{3-4}$. Nos últimos anos, pesquisadores têm despertado interesse em estudar este tema ${ }^{5-7}$. Estudos apontam prevalências de insatisfação corporal variando de $50 \%$ a $60 \%$ em adolescentes ${ }^{7-8}$. Entretanto, parece que estas prevalências são menores em adolescentes do sexo masculino ${ }^{9-10}$.

Adolescência é um período compreendido dos 10 aos 19 anos de idade segundo a Organização Mundial de Saúde ${ }^{11}$. Nesta etapa ocorrem diversas modificações corporais. As mais comuns no sexo masculino são: aumento gradativo da massa magra, estirão de crescimento da estatura e redução gradativa de gordura corporal ${ }^{12}$. Estas alteraçôes são reguladas pelo processo maturacional ${ }^{13-14}$. Parece que algumas destas mudanças corporais corroboram o ideal de beleza sociocultural masculino ao remeter-se o aumento da musculosidade 7 . Deste modo, acredita-se que o processo de maturação biológica proporcione adaptaçōes positivas na imagem corporal em meninos.

No que diz respeito aos jovens atletas, estes costumam apresentar maior desenvolvimento de massa magra quando comparados aos não atletas ${ }^{15-16}$. Talvez, por isso, alguns autores têm encontrado menor depreciação com a aparência corporal neste público $^{5,16}$. Por outro lado, McGeheE et al. ${ }^{17}$ e Filaire et $\mathrm{al}^{2}{ }^{2}$ salientam que o âmbito competitivo pode ser repleto de cobranças de treinadores, pais e amigos no anseio por melhores resultados. Sendo assim, o atleta pode estar mais vulnerável ao descontentamento com o corpo, caso não atinja o rendimento esportivo esperado $^{18}$. De qualquer forma, os resultados de pesquisas de imagem corporal têm sido residuais e inconclusivos a respeito de efeitos da morfologia do corpo sobre a insatisfação com o peso e a aparência física, principalmente na população de atletas ${ }^{6,19}$.

Por conseguinte, somente quatro estudos a respeito da insatisfação corporal foram encontrados no 
Brasil com jovens atletas do sexo masculino ${ }^{4,16,20-21}$. Ademais, não se identificou nenhuma pesquisa que tenha utilizado a maturação biológica como variável independente ou explicativa para a insatisfação

\section{Método}

\section{Amostra}

Trata-se de uma pesquisa transversal, com características correlacionais e comparativas, realizada no ano de 2011 nas cidades do Rio de Janeiro/RJ, Três Rios/ RJ, Juiz de Fora/MG e Barbacena/MG com atletas adolescentes do sexo masculino. Segundo o Comitê Olímpico Brasileiro, esta população no ano de 2010 era próxima a 15000 sujeitos. Por conseguinte, realizou-se cálculo amostral utilizando os seguintes critérios: prevalência de insatisfação corporal de 30\% segundo recomendações de dois estudos ${ }^{4,16} ; 5 \%$ de erro amostral com $95 \%$ de confiança, utilizando efeito de desenho de 1,4, totalizando 316 indivíduos que deveriam ser avaliados para representatividade da população. Foram incluídos na pesquisa atletas com idades entre 10 e 19 anos, que praticassem treinamento sistematizado de sua respectiva modalidade esportiva por pelo menos três vezes semanais com duração de uma hora por sessão, além de terem participado de competição no ano de 2011. Deste modo, 486 jovens atletas foram avaliados. No entanto, 22 sujeitos foram excluídos do estudo por não responderem o questionário em sua totalidade, ou por não participarem das aferições antropométricas. Ao todo, sete clubes participaram da pesquisa. As coletas de dados somente foram desenvolvidas após o projeto ser aprovado pelo Comitê de Ética e Pesquisa em Seres Humanos da Universidade Federal de Juiz de Fora.

\section{Instrumentos}

\section{Insatisfação corporal}

Foi avaliada por meio do Body Shape Questionnaire (BSQ). Consta de uma ferramenta de autopreenchimento composta por 34 perguntas em escala do tipo Likert, variando de $1=$ nunca até $6=$ sempre, que busca avaliar a frequência de preocupação/descontentamento que o jovem possui com o peso e a aparência física. Quanto maior o escore, maior é a depreciação que o avaliado tem com a sua aparência corporal. É possível, ainda, discriminar os corporal em adolescentes. Diante disso, o objetivo do presente estudo foi investigar se a maturação biológica influencia os níveis de insatisfação corporal em jovens atletas do sexo masculino.

respondentes em quatro níveis: livre de insatisfação corporal $(<80)$, leve insatisfação $(\geq 80 \mathrm{ou}<110)$, insatisfação moderada $(\geq 110$ ou $<140)$ e grave insatisfação com o corpo $(\geq 140)$. Este questionário foi validado para a população adolescente brasileira ${ }^{1}$, mostrando boas propriedades psicométricas. Para a presente amostra, foi calculada a consistência interna pelo alpha de Cronbach, obtendo-se valor de 0,92.

\section{Maturação biológica}

Foi avaliada por intermédio da maturação somática. Para este método é necessário aferir peso, estatura e altura tronco-cefálica. O comprimento de pernas é obtido pela diferença entre estatura e altura tronco-cefálica. Estas medidas, juntamente com a idade cronológica são utilizadas em uma equação estabelecida por MiRWALD et al. ${ }^{13}$, que estima a idade de pico de velocidade de crescimento em estatura. A fórmula nos fornece valores positivos e negativos, podendo classificar o sujeito da seguinte maneira ${ }^{22}$ :

$<-1=$ Pré estirão de crescimento em estatura;

$\geq-1$ ou $\leq+1=$ Durante o estirão de crescimento em estatura;

$>+1=$ Pós estirão de crescimento em estatura.

\section{Antropometria}

Massa corporal foi aferida por uma balança digital portátil da marca Tanita com precisão de 0,1 $\mathrm{kg}$ e capacidade máxima de $200 \mathrm{~kg}$. A estatura foi mensurada por um estadiômetro portátil da marca Tonelli com precisão de $0,5 \mathrm{~cm}$ e altura máxima de 2,20 m. Obteve-se o índice de massa corporal (IMC) pela razão entre massa corporal (kg) dividido pelo quadrado da estatura $(\mathrm{m})$. As dobras cutâneas (triciptal e subescapular) foram medidas por um plicômetro científico da marca Lange com precisão de $0,1 \mathrm{~mm}$. Utilizaram-se os procedimentos e padronizações estabelecidos pela ISAK ${ }^{23}$ para tais avaliaçōes. A altura tronco-cefálica foi aferida por um banco adaptado de madeira, construído segundo recomendaçôes de MiRWALD et al. ${ }^{13}$. 
As dobras cutâneas foram realizadas em triplicata de forma não consecutiva. Para estimar o percentual de gordura $(\% \mathrm{G})$ de atletas com idade entre $10 \mathrm{e}$ 17 anos, utilizou-se a equação de predição proposta por SLAUghter et al. ${ }^{24}$, que leva em consideração a etnia (branco ou negro) e a etapa maturacional em função da idade cronológica (pré-púbere - sete a 10 anos; púbere - 11 a 14 anos; e pós-púbere - 15 a 17 anos) do avaliado. Neste sentido, a etnia foi determinada por meio de autoavaliação. Nos atletas com idades iguais ou superiores a 18 anos, aferiram-se as dobras cutâneas peitoral, abdominal e coxa média, seguindo-se o padrão descrito pelos autores das equações preditivas em questão ${ }^{25}$. A densidade corporal foi obtida pelas equaçõos quadráticas de três dobras cutâneas de JACKSON e POLLOCK ${ }^{25}$, seguidas pela equação de $\operatorname{SIRI}^{26}$ para estimativa da porcentagem de gordura corporal. Todas estas medidas foram aferidas pelo mesmo avaliador com propósito de aumento da fidedignidade da avaliação. Contudo, as medidas ainda ficaram sujeitas a variabilidade intra-avaliador. A literatura recomenda que não ocorram diferenças superiores a $10 \%$ entre uma medida e as demais no mesmo local. Sendo assim, realizou-se o cálculo do Erro Técnico de Medida, proposto por SiLVA et al. ${ }^{27}$, excluindo dados com variância maior que $10 \%$.

\section{Procedimentos}

Primeiramente, os pesquisadores buscaram os diretores e responsáveis das equipes avaliadas na pesquisa para explanar os objetivos e procedimentos necessários para o presente estudo. Após o consentimento dos mesmos, marcou-se reunião com as equipes para explicar novamente objetivos e procedimentos da pesquisa. Neste encontro, foi entregue aos atletas, o termo de consentimento livre e esclarecido, pedindo-lhes que devolvessem assinado pelo responsável na semana subsequente.

$\mathrm{O}$ estudo foi dividido em dois momentos. $\mathrm{O}$ primeiro foi destinado à aplicação do BSQ. Esta etapa foi conduzida em grupo. Os questionários foram entregues aos participantes que receberam a mesma orientação verbal. Eventuais dúvidas foram esclarecidas por um único pesquisador a fim de evitar vieses e possíveis interferências externas. Não houve limite de tempo para preenchimento do BSQ.

Por conseguinte, o momento dois destinou-se a aferição das medidas antropométricas. Estes procedimentos foram realizados em uma sala separada disponibilizada pelos próprios clubes. Estas medidas foram mensuradas individualmente, permitindo-se outro atleta adentrar no ambiente, somente quando seu colega de equipe já tivesse se retirado do recinto.

\section{Análise dos dados}

O teste Kolmogorov Smirnov foi conduzido para averiguar a normalidade da distribuição dos dados. Como a distribuição dos dados não foi violada utilizaram-se medidas de tendência central (média e desvio padrão) para descrever variáveis contínuas e frequência (absoluta e relativa) para descrever variáveis dicotômicas. Conduziu-se a análise de covariância univariada (Ancova) para comparar algumas variáveis da investigação (BSQ, IMC e percentual de gordura) em função das classificações de maturação somática (pré-estirão, durante estirão e pós-estirão). Salienta-se que a variável "idade cronológica" foi controlada (inserida como covariável) nestas análises. Todas as análises foram realizadas no "software" SPSS 17.0, adotando-se nível de significância de 5\%.

\section{Resultados}

No total, participaram do estudo 464 atletas com média de idade de 15,05 ( $\pm 2,02)$ anos. As médias para IMC e percentual de gordura foram $21,28( \pm 2,90)$ e $18,43( \pm 7,26)$, respectivamente. A respeito da insatisfação corporal, somente $14,5 \%$ dos esportistas demonstraram depreciações com o próprio corpo (TABELA 1). As distribuiçōes de prevalência das classificações do BSQ e da amostra segundo modalidades esportivas estão apresentadas na TABELA 1. Em adição, os achados apontaram que $17,3 \%$ dos atletas do grupo Pré estirão, 15,1\% dos esportistas com classificação Durante estirão e $13,2 \%$ dos sujeitos do grupo Pós estirão estavam insatisfeitos com o peso e a aparência física. 
TABELA 1 - Distribuição de frequência (\%) de atletas adolescentes competitivos brasileiros por modalidade.

$\mathrm{IBSQ}=$ Body Shape Questionnaire;

$\mathrm{N}=$ Tamanho amostral.

\begin{tabular}{lcc}
\hline & Insatisfação corporal \\
\hline BSQ & N & Percentual (\%) \\
Livre de insatisfação corporal & 396 & 85,5 \\
Leve insatisfação & 49 & 10,6 \\
Moderada insatisfação & 13 & 2,8 \\
Grave insatisfação & 6 & 1,3
\end{tabular}

\begin{tabular}{lcc}
\hline & Modalidade esportiva & \\
\hline Nataçáo & 42 & 9,1 \\
Futebol & 271 & 58,4 \\
Handebol & 16 & 3,4 \\
Basquetebol & 56 & 12,1 \\
Voleibol & 6 & 1,3 \\
Atletismo & 10 & 2,2 \\
Triathlon & 14 & 3,0 \\
Judô & 14 & 3,0 \\
"Taek-won-do" & 6 & 1,3 \\
Esgrima & 8 & 1,7 \\
Pólo Aquático & 16 & 3,4 \\
Saltos Ornamentais & 5 & 1,1 \\
\hline Total & 464 & 100,0 \\
\hline
\end{tabular}

Em relação à comparação de insatisfação corporal, IMC e percentual de gordura de acordo com as classificações da maturação somática, existem alguns resultados que merecem destaque (TABELA 2). Não se evidenciaram diferenças estatisticamente significativas para as pontuações do BSQ. Por outro lado, valores de IMC de atletas classificados como Pré estirão foram menores quando comparados com os grupos Durante estirão e Pós estirão $(\mathrm{p}<0,05)$. No entanto, o percentual de gordura do grupo Pré estirão somente foi diferente da classificação Durante estirão $(\mathrm{p}<0,05)$, não identificando-se diferença estatística para sujeitos que já haviam passado do pico de estirão de crescimento em estatura, conforme demonstrado na TABELA 2.

TABELA 2 - Média e desvio padrão da insatisfação corporal segundo classificações de maturação somática pela análise univariada de covariância em jovens atletas do sexo masculino.

CMS = Classificação maturação somática; $\mathrm{DP}=$ Desvio padrão; $B S Q=$ Body Shape Questionnaire; IMC = Índice de massa corporal;

$\% \mathrm{G}=$ Percentual de gordura.

${ }^{b} p<0,05$ em relação ao grupo "Durante estirão"; " $p<0,05$ em relação ao grupo "Pós estirão".

\begin{tabular}{lccc}
\hline \multirow{2}{*}{ CMS } & Pré estirão $(\mathbf{n}=\mathbf{5 2})$ & Durante estirão $(\mathbf{n}=\mathbf{1 9 2})$ & Pós estirão $(\mathbf{n}=\mathbf{2 2 0})$ \\
& Média $( \pm \mathrm{DP})$ & Média $( \pm \mathrm{DP})$ & Média $( \pm \mathrm{DP})$ \\
\hline BSQ & $61,90( \pm 4,11)$ & $58,03( \pm 1,89)$ & $60,72( \pm 2,15)$ \\
$\mathrm{IMC}$ & $18,98( \pm 0,49)^{\mathrm{b}, \mathrm{c}}$ & $20,43( \pm 0,22)^{\mathrm{c}}$ & $22,57( \pm 0,25)$ \\
$\% \mathrm{G}$ & $20,52( \pm 0,69)^{\mathrm{b}}$ & $16,34( \pm 0,60)$ & $17,29( \pm 1,32)$ \\
\hline
\end{tabular}




\section{Discussão}

O presente estudo teve como premissa investigar a influencia da maturação biológica na insatisfação corporal de jovens atletas do sexo masculino. Os resultados evidenciaram baixa prevalência de insatisfação corporal entre os jovens atletas avaliados. Ademais, não foram encontradas diferenças de insatisfação corporal entre as classificações de maturação somática.

Os resultados da presente pesquisa demonstraram baixa proporção de atletas com descontentamento com o peso e a aparência corporal, corroborando outros achados ${ }^{4,18,20,28}$. Entretanto, alguns estudos têm apresentado resultados contrastantes, pois evidenciaram alta prevalência de insatisfação corporal no público de atletas do sexo masculino ${ }^{3,15}$. Talvez, estas discrepâncias de achados estejam relacionadas aos tipos de esportes avaliados ou a diferenças socioculturais entre os países. Pesquisadores ressaltam que atletas praticantes de esportes que enfatizam características estéticas ou mesmo os que possuem divisão por classe de peso ou ainda os que apresentam vestimentas que salientam o formato corporal, podem demonstrar maior prevalência de preocupação com a aparência física quando comparados a esportistas de outras modalidades ${ }^{4,15}$. Ademais, poucas pesquisas utilizaram o mesmo instrumento (BSQ) para avaliar a insatisfação corporal em atletas. Deste modo, fica impossibilitado comparar tais prevalências entre os estudos.

A respeito da comparação da insatisfação corporal segundo as classificações de maturação somática, a análise não evidenciou diferença estatística. Por outro lado, os meninos que não haviam atingido o pico de estirão de crescimento, juntamente com os que estavam passando por esta etapa maturacional apresentaram valores estatisticamente menores de IMC em relação ao grupo Pós estirão. Além disso, esportistas biologicamente mais jovens estavam com maior perfil percentual de gordura corporal quando comparados ao grupo Durante estirão. Conforme evidenciado, Siervogel et al. ${ }^{12}$ salientam que o processo maturacional no sexo masculino induz a redução do percentual de gordura, bem como o aumento gradual de peso corporal, que por sua vez, é associado ao crescimento da massa muscular. Acrescentando, BAXTER-JONES et al. ${ }^{14}$ ressaltam que estas alterações morfológicas são oriundas da acentuação da secreção do hormônio testosterona pela hipófise anterior durante o período pubertário.

Os achados indicaram que a depreciação com o peso e a aparência corporal foi semelhante em diferentes estágios maturacionais em jovens atletas do sexo masculino, independente das características morfológicas. FORTES e FERREIRA ${ }^{4}$ argumentam que meninos esportistas costumam não apresentar acentuada insatisfação com o corpo, independente da fase da adolescência. Talvez os atletas masculinos estejam mais preocupados com o desempenho esportivo do que com as suas características morfológicas ${ }^{6,16}$. Por outro lado, ARroYo et al. ${ }^{19}$ afirmam que jovens atletas, independente do sexo, podem estar vulneráveis para internalizar padrões socioculturais de beleza. Deste modo, os atletas podem almejar músculos maiores e menor percentual de gordura ${ }^{17}$. Sendo assim, são necessárias mais pesquisas a respeito deste tópico, a fim de esclarecer melhor as influências biológicas a partir das comparações de insatisfação corporal segundo maturação biológica, de preferência isolando efeitos de fatores culturais.

Cabe ressaltar que a insatisfação corporal de jovens atletas pode sofrer influência de outros fatores. Por exemplo, as pressões para a otimização do desempenho esportivo exercida por treinadores, pais e amigos. Pesquisadores salientam que as cobranças para a melhora do rendimento atlético direcionadas aos atletas podem torna-los mais insatisfeitos com o corpo, caso não atinjam o resultado competitivo esperado ${ }^{2}$.

Os achados do presente estudo são importante sno que diz respeito a uma primeira identificação de como jovens atletas se percebem quanto a sua insatisfação corporal, considerando as diferentes etapas de maturacionais, no entanto, a pesquisa apresentou algumas limitações que merecem ser destacadas. Primeiramente, utilizou-se instrumento autorreportado para avaliar a insatisfação corporal. Pesquisadores argumentam que este tipo de ferramenta pode não trazer resultados confiáveis por tratar-se de respostas subjetivas, ainda mais com a população de atletas, que podem temer corte da equipe dependendo dos resultados encontrados. Por outro lado, Sundgot-Borgen e Torstveit ${ }^{15}$ recomendam o uso de questionários em pesquisas com tamanho amostral grande, por conta da facilidade de sua aplicabilidade, além do baixo custo operacional. Ademais, pode-se considerar o corte transversal como outra limitação do estudo, pois o mesmo não permitiu que fosse realizada inferência de causalidade. Isso significa que não se tem como avaliar o grau de intensidade e a direção das associações encontradas entre o desfecho do estudo e a variável independente. $\mathrm{Na}$ realidade, esta é uma limitação do estudo transversal, por apresentar um 
retrato instantâneo do contexto avaliado. No mais, entende-se que os achados deste estudo possam acrescentar informações importantes a literatura científica que merecem ser comparados e discutidos em outras pesquisas. Os resultados permitiram concluir que a insatisfação com o corpo foi semelhante entre as diferentes etapas maturacionais em jovens atletas do sexo masculino.

Recomenda-se o desenvolvimento de estudos longitudinais com propósito de avaliar a mudança da imagem corporal de acordo com as alteraçóes morfológicas durante o período da adolescência.

\begin{abstract}
Body dissatisfaction and biology maturation in male athletes

The aim of this study was to investigate the influence of biological maturation on body dissatisfaction (BD) of young male athletes. 464 athletes participated in the survey. The BD was measured due to the Body Shape Questionnaire (BSO). The biological maturity was estimated by somatic maturation. Weight, height and skinfold thickness were measured to calculate body mass index (BMI) and estimate the percentage of fat, respectively. Statistical tests were conducted in specialized software to analyze the data. The results showed no statistically significant differences for the scores of the BSO according to ratings somatic maturation. Moreover, we identified differences in $\mathrm{BMI}$ and body fat percentage between maturational stages ( $\mathrm{p}$ $<0.05$ ). Thus, it was concluded that the BD of young athletes was not influenced by biological maturation.
\end{abstract}

KEY WORDS: Body image; Athletes; Sport.

\title{
Referências
}

1. Conti MA, Cordás TA, Latorre MRDO. Estudo de validade e confiabilidade da versão brasileira do bodyshapequestionnaire (bsq) para adolescentes. Rev Bras Saude Mater Infant. 2009;9:331-8.

2. Filaire E, Rouveix M, Pannafieux C, Ferrand C. Eating atitudes, perfectionism and body-esteem of elite male judoists and cyclists. J Sports Sci Med. 2007;6:50-7.

3. Hallsworth L, Wade T, Tiggeman M. Individual differences in male body image: an examination of self-objectification in recreational body builders. Br J Health Psychol. 2005;10:453-65.

4. Fortes LS, Ferreira MEC. Comparação da insatisfação corporal e do comportamento alimentar inadequado em atletas adolescentes de diferentes modalidades esportivas. Rev Bras Educ Fís Esporte. 2011;24:707-16.

5. Haunsenblas HA, Downs DS. Comparison of body image between athletes and nonathletes: a meta-analytic review. J Appl Sport Psychol. 2001;13:323-39.

6. Toro J, Galilea B, Martinez-Mállen E, et al. Eating disorders in Spanish female athletes. Int J Sports Med. 2005;26:693-700.

7. Mousa T, Marshal RH, Al-Domi HA, Jibril MA. Body image dissatisfaction among adolescent schoolgirls in Jordan. Body Image. 2010;7:46-50.

8. Alves E, Vasconcelos FAG, Calvo MCM, Neves J. Prevalência de sintomas de anorexia nervosa e insatisfação com a imagem corporal em adolescentes do sexo feminino do Município de Florianópolis, Santa Catarina, Brasil. Cad Saúde Pública. 2008;24:503-12.

9. Castro IRR, Levy RB, Cardoso LO, et al. Imagem corporal, estado nutricional e comportamento com relação ao peso entre adolescentes brasileiros. Ciênc Saúde Colet. 2010;15:3099-108. Suppl.2

10. Matias TS, Rolim MKSB, Kretz FL, Schmoelz CP, Andrade A. Satisfação corporal associada a prática de atividade física na adolescência. Motriz. 2010;16:370-8.

11. World Health Organization (WHO).Development of a WHO growth reference for school-aged children and adolescents. Bull World Health Org. 2007;85:660-7.

12. Siervogel RM, Demerath EW, Schubert C, et al. Puberty and body composition. Horm Res. 2003;60:36-45.

13. Mirwald RL, Baxter-Jovens ADG, Bailey DG, Bernen GP. An assessment of maturity from anthropometric measurements. Med Sci Sports Exerc. 2002;34:689-94. 
14. Baxter-Jones D, Eisenmann JC, Sherar LB. Controlling for maturation in Pediatric exercise science. Pediatr Exerc Sci. 2005;17:18-30.

15. Sundgot-Borgen J, Torstveit MK. Aspects of disordered eating continuum in elite high-intensity sports. Scand J Med Sci Sports. 2010;20(Suppl 2):112-21.

16. Fortes LS, Miranda VPN, Amaral ACS, Ferreira MEC. Insatisfação corporal de adolescentes atletas e não atletas. J Bras Psiquiatr. 2011;60:309-14.

17. McGehee TMT, Green JM, Leeper JD, Leaver-Dunn D, Richardson M, Bishop PA. Body image, anthropometric measures, and eating-disorder prevalence in auxiliary unit members. J Athl Train. 2009;44:418-26.

18. Costarelli V, Stamou D. Emotional intelligence, body image and disordered eating attitudes in combat sport athletes. J Exerc Sci Fit. 2009;7:104-11.

19. Arroyo M, Gonzalez-De-Suco JM, Sanchez C, Ansotegui L, Rocandio AM. Body image and body composition: comparisons of young male elite soccer players and controls. Int J Sport Nutr Exerc Metab. 2008;18:628-38.

20. Vieira JLL, Oliveira LP, Vieira LF, Vissoci JRN, Hoshino EF, Fernandes SL. Distúrbios de atitudes alimentares e sua relação com a distorção da auto-imagem corporal em atletas de judô do Estado do Paraná. Rev Educ Fís/UEM. 2006;17:177-84.

21. Fortes LS, Matta MO, Paes ST, Ferreira MEC. Fatores de risco associados ao comportamento alimentar inadequado em futebolistas. Rev Bras Educ Fís Esporte. 2012;26:447-54.

22. Fortes LS, Almeida SS, Ferreira MEC. Processo maturacional, insatisfação corporal e comportamento alimentar inadequado em jovens atletas. Rev Nutr. 2012;25:575-86.

23. The Internacional Society for Advancement for Kineanthropometry (ISAK). Sidney: National Library of Australia; 2001.

24. Slaughter MH, Lohman TG, Boileau R, Hoswill CA, Stillman RJ, Yanloan MD. Skinfold equations for estimation of body fatness in children and youth. Hum Biol. 1988;60:709-23.

25. Jackson AS, Pollock ML. Generalized equations for predicting body density of men. Br J Nutr. 1978;40:497-504.

26. Siri WE. The gross composition of the body. In: Tobias CA, Lawrence JH, editors. Advances in biological and medical physics. New York: Academic Press; 1956. p. 239-80.

27. Silva DAS, Pelegrini A, Pires-Neto CS, Vieira MFS, Petroski EL. O antropometrista na busca de dados mais confiáveis. Rev Bras Cineantropom Desempenho Hum. 2011;13:82-5.

28. Peden J, Stiles BL, Vandehey M, Diekhoff G. The effects of external pressures and competitiveness on characteristics of eating disorders and body dissatisfaction. J Sport Social Issues. 2008;32:415-29.

\begin{tabular}{r|l} 
ENDEREÇO & \\
Leonardo de Sousa Fortes & \\
Universidade Federal de Juiz de Fora & Recebido para publicação: 28/03/2012 \\
R. Hamelleto Fellet, 20/201 & 1a. Revisão: 15/11/2012 \\
36036-130 - Juiz de Fora - MG - BRASIL & 2a. Revisão: 27/03/2013 \\
e-mail: leodesousafortes@hotmail.com & Aceito: 28/03/2013
\end{tabular}

